\title{
Identifying of Noise Type and Estimating of Noise Level for InSAR Interferogram in the Wavelet Domain
}

\author{
Cai Guolin, Liu Guoxiang, Zhang Aoli, Sun Meiling, Tang Jiafa, Zhang Shuaiyi \\ (Dept. of RS and GIS, Southwest Jiaotong University, Chengdu 610031, China)
}

\begin{abstract}
In order to identify noise type of the InSAR interferogram and facilitate more effective interferogram processing, especially for interferogram filtering based on wavelet transform, a novel methodology for identifying noise type and estimating noise level was reported. This methodology combined a wavelet transform algorithm with a logarithmic transform algorithm, and carried on the thorough analysis for the characteristics of interferogram wavelet coefficients from two aspects of energy distribution and statistics. Firstly, energy distribution characteristics of low frequency son-band coefficients were researched before and after the logarithmic transform, so that noise type of interferogram can be identified. On this base, the noise level would be estimated by high frequency coefficients. From the analysis results obtained in this study, the conclusions can be made that noise of InSAR interferogram is the additive noise, high frequency coefficients show standard normal distribution, and the noise level could be estimated by HH son-band on one scale. These results provide more spaces for noise analysis and processing of InSAR interferogram based on the wavelet transform algorithm.
\end{abstract}

Key words: InSAR Interferogram, Noise, Wavelet Coefficient, Energy

\section{I . INTRODUCTION}

InSAR can distill ground 3D information and ground deformation information mainly by using interferometric phase information which can be extracted with many SAR images acquired over the same region ${ }^{[1,2]}$. In the process of InSAR data, phase unwrapping is very important. However, due to the effect of several factors, such as quality of the SAR image, time decorrelation, geometry decorrelation, etc., the interferograms are polluted by the noises, and these noises increase the difficulty of phase unwrapping. This may cause a failure in phase unwrapping ${ }^{[3,4]}$. So, before phase unwrapping, how to improve the interferogram quality becomes the key problem to the filtering process.

At present, there are many methods of InSAR interferogram filtering, not only in space domain ${ }^{[5,6]}$, but also in frequency domain [7]. But these applications mostly transplant filtering-algorithms of general images and visible remote sensing images to InSAR interferogram without the analysis of noise characteristics. Although Lee ${ }^{[8]}$ has proved that the interferometric phase noise is additive, still many researchers consider that the interferogram contains multiplicative noise (speckle noise). That is subjective speculation or assumption of related prior knowledge for noise, and don't rely on objective statistics and analysis, the filtering in this case is often blind. As we may think, if a filtering algorithm is applied to a "mistake" interferogram (the

Fund Projects: The work of this paper is supported by National Basic Research Program of China (973) (No. 2012CB719901), National Natural Science Fund Item (No. 41074005, 41271448, 41201434), the Fundamental Research Funds for the Central Universities (No. 2682013CX009, SWJTU09ZT01,

SWJTU10ZT02, SWJTU11CX139, SWJTU11ZT13, SWJTU09BR015), Item of China Ministry of Education (No. 12YJAZH124). noise type, distribution, etc. are different from assumptions), the results could be imagined ${ }^{[9]}$. Therefore, before InSAR interferogram filtering, an in-depth analysis of noise type and distribution characteristics is very necessary.

For this purpose, we present a novel methodology of identifying noise type. This methodology is based on wavelet transforms and logarithmic transforms, and identifies noise types from energy and distribution characteristics. Distribution rules of the noise are analyzed and its level is estimated by this methodology. In order to test the reliability of the results, the experiment will also be compared with the results of the noises known images.

\section{II . IDENTIFYING METHOD FOR NOISE TYPE OF INTERFEROGRAM BASED ON WAVELET DOMAIN}

\section{A. Wavelet Transform and Impact Analysis of Noise}

The main idea of wavelet transform as follows ${ }^{[10]}$ : Firstly, $L^{2}(R)\left(L^{2}(R)\right.$ is the collectivity of a square integrable function in real number set) is decomposed into a series of subspace sequence with different resolution. Secondly, object $S$ is described in $L^{2}(R)$ as the imminent limit of a series of approximate functions, and each approximate function is the projection of $S$ in different resolution subspaces. Finally, the configuration and character of $S$ in the different resolution subspace are analyzed through these projections.

The analysis of image noise using the characteristics of wavelet transforms is a better method ${ }^{[11]}$. According to the relationship between the noise and the signal, the noise can be divided into additive noise and multiplicative noise. Between them, the mathematical model of additive noise is:

$$
P(x, y)=S(x, y)+N(x, y)
$$

In the formula, $P(x, y)$ is for image with noise, $S(x, y)$ is for signal without noise, and $N(x, y)$ is for noise. And the mathematical model of multiplicative noise is:

$$
P(x, y)=S(x, y) \times N(x, y)
$$

According to the image with additive noise $P(x, y)$, the orthogonal wavelet transform operator is set as $W$. The wavelet coefficient matrix and the relationship of $P(x, y)$ after wavelet transform can be expressed as:

$$
\begin{array}{cl}
Y=W \bullet P & X=W \bullet S \quad V=W \bullet N \\
Y & =X+V
\end{array}
$$

Wavelet coefficients of the image with additive noise are also additive, but the image with multiplicative noise does not have this characteristic. In addition, after a wavelet transform of the image with additive noise, the energy of image 
(concepts seen also literature [12]) are mainly concentrated in the low frequency son-band. High frequency son-band coefficients have small value and low energy, and the noise mainly reflects in the high frequency son-band (especially the diagonal son-band) ${ }^{[9]}$. So, for an image, even be affected by additive noise, but after wavelet decomposition, the wavelet coefficient's energy in low frequency still won't have too big change.

For images with multiplicative noise, because it does not have additives, after direct wavelet decomposition, noise and information are inseparable, which cause the wavelet coefficient's energy in low frequency of multiplicative noise great changes. That is because the influence of multiplicative noise, it makes the amplitude of low frequency son-band coefficients smaller, and then causes the proportion of the low frequency wavelet coefficient's energy in the total energy to be significantly reduced.

B. Identifying Method for Noise Type of Interferogram Based on Wavelet Transform and Logarithmic Transform

With the comprehensive analysis, we can conclude that by wavelet decomposition, most of the wavelet coefficient's energy concentrates on low frequency son-bands, and the influence of additive noise and multiplicative noise to the image wavelet coefficient's energy are different. Among them, the effect of additive noise takes place in the high frequency part, and the influence of multiplicative noise are in the low frequency part. This property makes the noise analysis using wavelet coefficients possible.

If the logarithmic conversions were carried out to interferogram with multiplicative noise, we can acquire the result:

$$
\bar{P}(x, y)=\bar{S}(x, y)+\bar{N}(x, y)
$$

Where $\bar{P}(x, y)=\ln (|P(x, y)|), \bar{S}(x, y)=\ln (|S(x, y)|)$, $\bar{N}(x, y)=\ln (|N(x, y)|)$. By formula (4), it can be seen that after the logarithmic conversion, the multiplicative operation of signal and noise is converted to added operations. Now, if the wavelet transform were carried out to the results of formula (4), the low frequency wavelet coefficients energy mainly reflect signal information. Accordingly, the proportion of energy in total energy will have significant improvement comparing with logarithmic conversion. But for image with additive noise, whether or not the logarithmic conversion is done, the proportion of low frequency wavelet coefficients energy in total will not change greatly.

Thus, according to the different characteristics of multiplicative noise and additive noise in the wavelet domain, from the angle of energy wavelet coefficients, we can propose an identification method of interferogram noise-type based on wavelet transform and logarithmic transform. The strategy of this method is as follows:

(1) Conduct wavelet transform to interferogram $P$ and calculate low frequency wavelet coefficients energy ratio of the total energy on every scale $V_{j}(j=1,2, \cdots, 7,8$, for wavelet decomposition scale).
(2) Conduct logarithmic transform to interferogram $P$, we can acquire $P_{l g}$.

(3) Use wavelet function to decompose $P_{l g}$ and calculate the low frequency wavelet coefficients' energy ratio of the total energy on every scale $V_{j}^{\prime}$.

(4) Compare $V_{j}$ with $V_{j}^{\prime}$, if $\left|V_{j^{-}} V_{j}^{\prime}\right|<=5 \%$ (two standard deviations corresponding confidence interval when error statistics) is present, it shows there are not any significant changes to the low-frequency coefficient's energy. Then we can assume the interferogram contains additive noise, instead of the interferogram containing multiplicative noise.

\section{IDENTIFICATION AND ANALYSIS OF INTERFEROGRAM NOISE}

\section{A. Experiment Data}

Based on the scheme mentioned in last section, we write the corresponding noise type identification procedures based on wavelet transform and logarithmic conversion. To test the validity of this method and identify InSAR interferogram noise type, this study choose two kinds of $\mathrm{C}$ band real interferograms of America Phoenix area with high signal-to-noise ratio and Iran Bam low signal-to-noise ratio (respectively called Bam_p and Pho_p for short) as the experiment data. And the interceptions of two interferograms are $800 * 1000$ pixels. Table 1 lists the satellite platform and imaging date.

Tab.1 Parameters of the interferograms for the experiment

\begin{tabular}{|c|c|c|c|}
\hline Video site & Video No. & $\begin{array}{c}\text { Satellite } \\
\text { platform }\end{array}$ & Imaging date \\
\hline \multirow{2}{*}{ Phoenix } & 1 & ERS1 & 1995.11 .05 \\
\cline { 2 - 4 } & 2 & ERS2 & 1995.11 .06 \\
\hline \multirow{2}{*}{ Bam } & 3 & ASAR & 2003.06 .11 \\
\cline { 2 - 4 } & 4 & ASAR & 2004.02 .11 \\
\hline
\end{tabular}

The experiment will also choose other noise known images as comparative data to verify the effectiveness of the proposed method and the correctness of estimating for InSAR interferogram noise type. Specifically: (1) a SAR image with the multiplicative noise (dujiangyan in China, platform for TSX1, x-band, 1m, time for May 15,2008, hereinafter referred to as DJY), interception size for $4800 * 4800$ pixel; (2) an aviation image (westconcordorthophoto.png, hereinafter referred to as WCOP), size of $366 * 364$ pixels, and add 0.2 zero-mean gaussian white noise and 0.2 speckle multiplicative noise to WCOP image, and generate two new pictures, respectively WCOP_g and WCOP_s. These above six images are shown in figure 1. Through comparative analysis of the data, we can explore what kind of noise pollutes InSAR interferogram and estimate its value. 


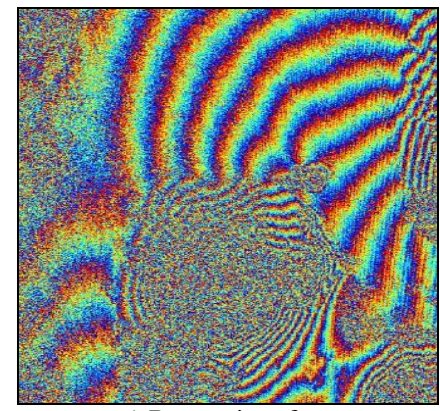

a) Bam_p interferogram

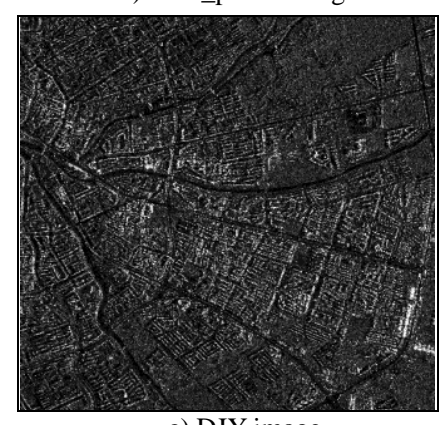

c) DJY image

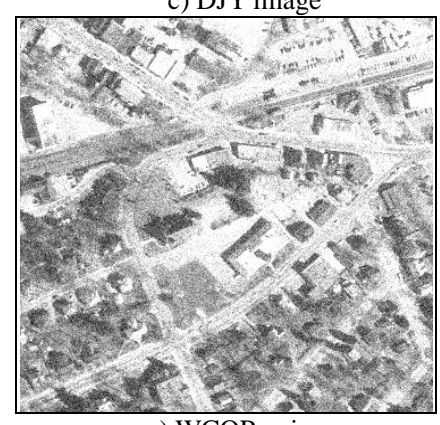

e) WCOP_g image

Fig.1 Six images for testing

B. Analysis of Energy Distribution to Interferogram Coefficients in Low Frequency

By section 2.1, it is known that after $N$ levels wavelet decomposition, image's energy distribute in $3 N+1$ son-band, and among them, LL for the low frequency son-band, the rest for high frequency son-band. Figure 2 a) for using bior6.8 wavelet (any wavelet operator can) to perform 8 scale wavelet decomposition with 6 images in figure 1 , gets the energy proportion of different scales of LL son-band. Figure $2 \mathrm{~b}$ ) for logarithmic conversion to 6 images first and 8 scale wavelet decomposition using wavelet bior6.8, get energy proportion of different scales LL son-band.

\begin{tabular}{|l|l|l|l|l|l|l|l|l|}
\hline & $\mathbf{1}$ & $\mathbf{2}$ & $\mathbf{3}$ & $\mathbf{4}$ & $\mathbf{5}$ & $\mathbf{6}$ & $\mathbf{7}$ & $\mathbf{8}$ \\
\hline Pho_p & 0.94 & 0.93 & 0.92 & 0.93 & 0.93 & 0.96 & 0.98 & 0.99 \\
& 72 & 2 & 82 & 07 & 5 & 36 & 25 & 61 \\
\hline DJY & 0.96 & 0.85 & 0.73 & 0.65 & 0.59 & 0.59 & 0.65 & 0.75 \\
& 94 & 85 & 92 & 77 & 93 & 85 & 48 & 77 \\
\hline WCOP & 0.99 & 0.98 & 0.96 & 0.95 & 0.95 & 0.96 & 0.98 & 0.99 \\
& 36 & 16 & 61 & 11 & 34 & 87 & 22 & 09 \\
\hline WCOP & 0.98 & 0.97 & 0.97 & 0.97 & 0.97 & 0.98 & 0.99 & 0.99 \\
_g & 68 & 97 & 43 & 01 & 48 & 48 & 21 & 64 \\
\hline WCOP & 0.89 & 0.86 & 0.87 & 0.88 & 0.92 & 0.95 & 0.98 & 0.99 \\
_s & 27 & 73 & 3 & 99 & 2 & 92 & 09 & 18 \\
\hline Bam_p & 0.89 & 0.87 & 0.88 & 0.89 & 0.92 & 0.94 & 0.96 & 0.98 \\
& 44 & 65 & 16 & 72 & 2 & 17 & 32 & 54 \\
\hline
\end{tabular}

a) The relationship between energy proportion of LL son-bond and wavelet decomposition scale (Without logarithmic conversion)

\begin{tabular}{|l|l|l|l|l|l|l|l|l|}
\hline & $\mathbf{1}$ & $\mathbf{2}$ & $\mathbf{3}$ & $\mathbf{4}$ & $\mathbf{5}$ & $\mathbf{6}$ & $\mathbf{7}$ & $\mathbf{8}$ \\
\hline Pho_p & 0.95 & 0.93 & 0.93 & 0.93 & 0.94 & 0.96 & 0.98 & 0.99 \\
& 09 & 74 & 46 & 78 & 36 & 56 & 57 & 89 \\
\hline DJY & 0.99 & 0.97 & 0.96 & 0.95 & 0.95 & 0.95 & 0.96 & 0.97 \\
& 24 & 68 & 4 & 59 & 01 & 28 & 33 & 73 \\
\hline WCOP & 0.99 & 0.99 & 0.99 & 0.99 & 0.99 & 0.99 & 0.99 & 0.99 \\
& 97 & 91 & 83 & 76 & 79 & 86 & 93 & 96 \\
\hline WCOP & 0.99 & 0.99 & 0.99 & 0.99 & 0.99 & 0.99 & 0.99 & 0.99 \\
_g & 93 & 9 & 88 & 86 & 89 & 94 & 97 & 99 \\
\hline WCOP & 0.99 & 0.99 & 0.99 & 0.99 & 0.99 & 0.99 & 0.99 & 0.99 \\
_S & 24 & 07 & 13 & 28 & 53 & 76 & 89 & 96 \\
\hline Bam_p & 0.90 & 0.89 & 0.89 & 0.91 & 0.93 & 0.95 & 0.97 & 0.99 \\
& 79 & 23 & 71 & 14 & 37 & 97 & 75 & 34 \\
\hline
\end{tabular}

b) The relationship between energy proportion of LL son-bond and wavelet decomposition scale (With logarithmic conversion)

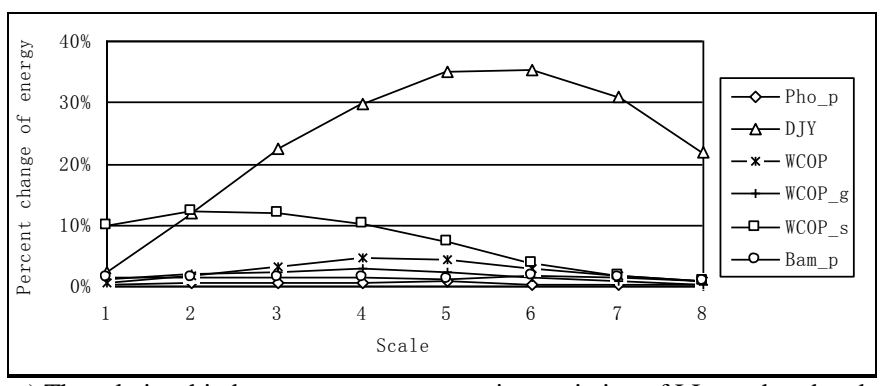

c) The relationship between energy proportion variation of LL son-bond and wavelet decomposition scale before and after logarithmic conversion Fig.2 Relation between the proportions of energy on LL son-bands and the scales of wavelet decomposition

Statistics in Figure 2 show that: (1) no matter whether logarithmic conversion operations are performed, LL son-band coefficients gather most of the energy, and the remaining little energy is distributed in all scales of high frequency son-band. (2) WCOP_s image and DJY image contain multiplicative noise, reflected in figure $2 \mathrm{c}$ ): convert the multiplicative operation to additive operation with logarithmic conversion, the proportion of LL son-band coefficients' energy are obviously improved, and DJY is increased by $35.43 \%$, WCOP_s improved $12.34 \%$. Both of them are far over the two standard deviations corresponding confidence interval of 5\%. (3) WCOP image doesn't contain noise, WCOP_g image contains only additive noise, after logarithmic conversions of the two images, the proportion of LL son-band coefficients energy has only small change. (4) Low frequency wavelet coefficients' energy distribution characteristics of Bam_p interferogram and Pho_p interferogram are the same as WCOP images, WCOP_g image. These changes are mainly caused by the algorithm error which can be ignored. (5) The energy of low frequency wavelet coefficients will not reduce with the scale increasing.

Through the above statistics and analysis, we can draw the following conclusions: (1) InSAR interferogram doesn't contain multiplicative noise but only additive noise. If it contains multiplicative noise, after the logarithmic conversion, the proportions of LL son-band coefficients' energy will change greatly. (2) By using the wavelet transform algorithm for image wavelet decomposition, the decomposition scale 
can be decided by energy proportion of LL son-band coefficients, and the minimum proportion corresponds to the best decomposition scale.

C. Estimation of Interferogram Noise Level Based on Coefficients in HH Son-band

Additive noise by wavelet decomposition is mainly distributed in high frequency son-bands to the first decomposition, and most obviously in the diagonal son-band, which can reflect the level of the noise ${ }^{[9]}$. Therefore, this paper researches the first decomposed diagonal son-band wavelet coefficients, analysis of the characteristics of noise in wavelet domain, and provides the reference for processing interferograms with noise, especially based on wavelet transforms.

In figure 1, we see statistics on the distribution of the one scale high frequency son-band coefficients of Bam_p, Pho_p, WCOP, and WCOP. Four images (the remaining two contain multiplicative noise, we will not talk about them), the results shown in figure 3.

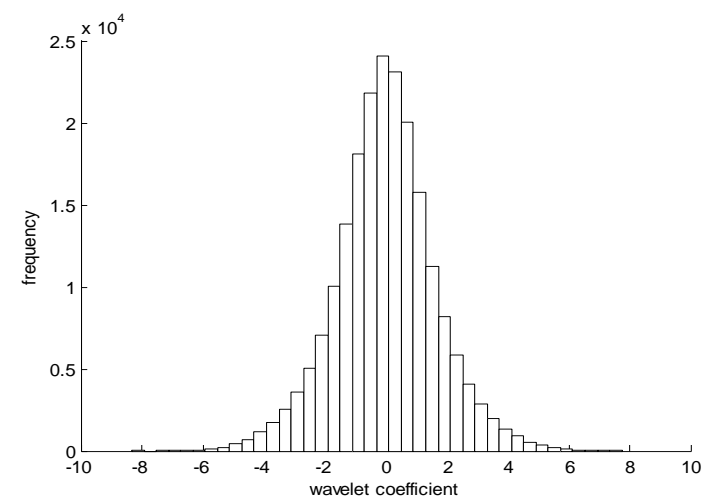

a) Bam_p

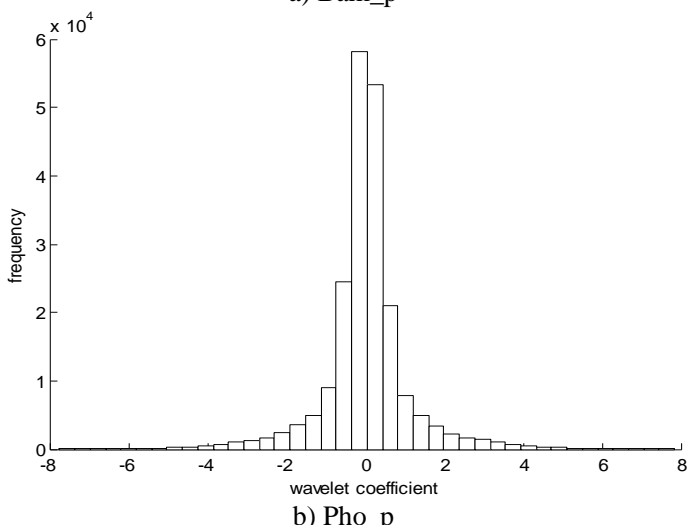

b) Pho_p

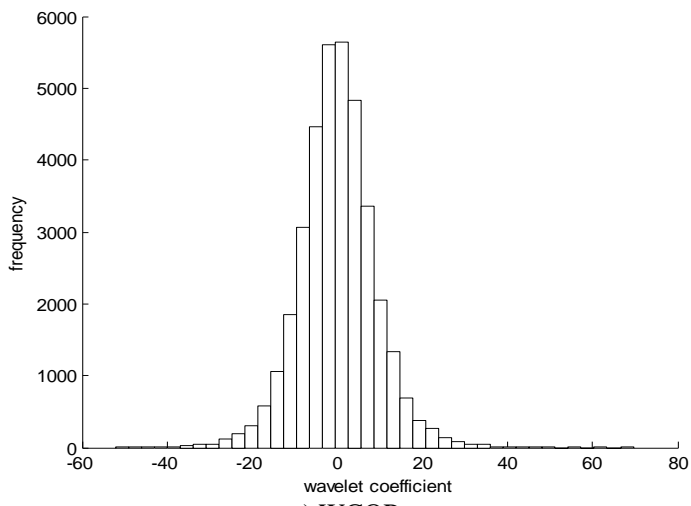

c) WCOP

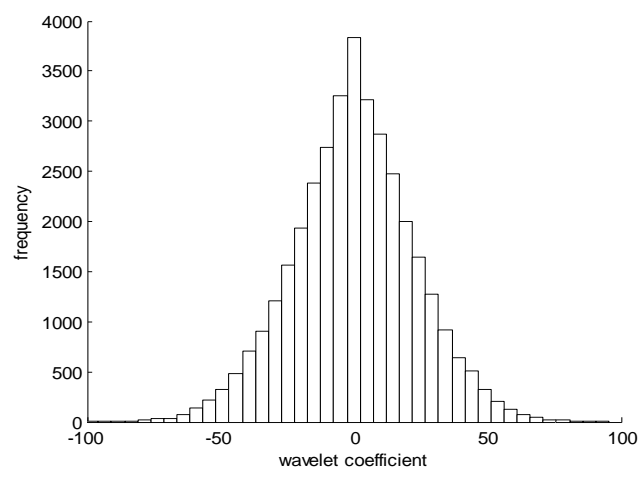

d) WCOP_g

Fig.3 Distribution map of diagonal HF coefficients on one scale for 4 images

From figure 3 , we can see that by wavelet transform, WCOP_g including additive gaussian white noise is similar to WCOP, subject to standard normal distribution, but the standard deviation becomes larger. Reflecting on Bam_p and Pho_p, they also are subject to standard normal distribution. Similarly, due to large Bam_p noise, its standard deviation is more than Pho_p.

Because wavelet coefficients are additive, it makes noise estimation using wavelet coefficients possible. The simplest method is to select the variance of high frequency $(\mathrm{HH})$ son-band coefficients as the variance of noise ${ }^{[3]}$. Donoho and Johnstone ${ }^{[13]}$ raised the estimation formula of noise variance:

$$
\sigma_{n}=\mid \text { median } \mid / 0.6745
$$

Among them, median is the mid-value wavelet coefficient's range in the first scale $\mathrm{HH}$ son-band. The method has been widely used in image denoising. Table 2 shows the wavelet domain noise variance statistic by the formula (5).

\begin{tabular}{|c|c|c|c|c|}
\multicolumn{1}{c}{ Table 2 noise variance estimation of the four images } \\
\hline & WCOP & WCOP_g & Bam_p & Pho_p \\
\hline $\boldsymbol{\sigma}$ & 0.03 & 0.18 & 0.17 & 0.05 \\
\hline
\end{tabular}

From table 2, we can see WCOP contains no noise, and its corresponding variance valuations are very small. WCOP_g valuations are approximately equal to the value of the added noise. The valuations of Bam_p and Pho_p are also in accordance with its own noise. This provides the following conclusion: after wavelet transform of InSAR interferogram including additive noise, the wavelet coefficients obey the standard normal distribution, and noise level can be estimated 
with the middle value of wavelet coefficients in $\mathrm{HH}$ son-band. This property has the certain reference to interferograms filtering based on wavelet domain.

\section{IV . SUMMARY}

This paper, which is based on the different effects of multiplicative noise and additive noise to the energy distribution of low frequency wavelet coefficients, proposes an identification method of the InSAR interferogram noise which is based on wavelet transform and logarithm transform. In order to test the effectiveness of the algorithm and the correctness of the InSAR interferogram noise-type identifying, this paper selects the $\mathrm{C}$ band InSAR interferogram from the Phoenix area's high signal-to-noise and the Bam area's low signal-to-noise to experiment, and then analyzes noise characteristics and noise level in wavelet domain based on the comparison with other images known noise. Through the contrast and analysis, the specific conclusions are as follows:

(1) The noise in the InSAR interferogram is the additive noise. SAR image contains a lot of speckle noise (multiplicative noise), but in the process of interferogram generated, multiplicative noise has been eliminated. At the same time, we can use the method in the paper to detect other images and whether they polluted by multiplicative noise.

(2) After the InSAR interferogram including additive noise, gets through the wavelet transform, high frequency coefficients obey standard normal distribution, and the greater the noise, the bigger the corresponding standard deviation. The noise value can be estimated by using $\mathrm{HH}$ son-band coefficients in the first scale. These characteristics have the certain references to the interferogram filtering based on wavelet domain.

\section{REFERENCES}

[1] Liu G. X., Ding X. L., Li Z. L., etal. Experimental Investigation on DEM Generation through InSAR [J]. Acta Geodaetica et Cartographica Sinica, 2001, 30(4): 337-343.

[2] Shan X. J., Song X. Y., Liu J. H., etal. Obtaining Digital
Elevation Data in Different Terrain and Physiognomy Regions with Spaceborne InSAR and Its Application Analysis [J]. Chinese Science Bulletin, 2002, 47(10): 868-873.

[3] Cai G. L., Liu G. X., Li Y. S. An Algorithm of Filtering InSAR Interferogram Based on Wavelet Phase Analysis [J]. Acta Geodaetica et Cartographica Sinica, 2008, 37(3): 293-300.

[4] Cai G. L., Li Y. S., Liu G. X. Wavelet-wiener Combined Filter and Its Application on InSAR interferogram [J]. Journal of Remote Sensing, 2009, 13(1): 129-136.

[5] Yu J. T., Chen Y. A New Filter on Interferogram Fringe [J]. Acta Geodaetica et Cartographica Sinica, 2004, 33(2): 121-126.

[6] Yuan Y. N., Hu Q. D., Mao S. Y. Denoising Methods in Interferometric SAR Images Processing[J]. Chinese Journal of Aeronautics, 1999, 12(2): 87-92.

[7] Wang L. C., Wang Y. N., Mao L. P. An Algorithm of Interferometric Phase Filter of InSAR Based on Wavelet Analysis and Median Filter Algorithm [J]. Acta Geodaetica et Cartographica Sinica, 2005, 34(2): 108-112.

[8] LEE J. S., Papathanassiou K. P., Ainsworth T., Grunes M. R., Reigber A. A New Technique for Noise Filtering of SAR Interferometric Phase Images [J]. IEEE Trans. Geosci. Remote Sensing, 1998, 36(1):1456-1465.

[9] Zhang Q., Liang D. Q., Fan X., etal. Idetifying of Noise Types And Estimating Of Noise Level For A Noisy Image In The Wavelet Domain [J]. Journal of Infrared and Millimeter Waves, 2004, 23(4): 281-285.

[10] Mallat S. Theory from Multi-resolution Signal Decomposition: the Wavelet Representation [J]. IEEE Transactions on Pattern Analysis and Machine Intelligence, 1989, 11(7): 674-693.

[11] Gao Q. W., Zhao Y. F., Lu Y. X. Despeckling SAR Images Using Stationary Wavelet Transform Combining with Directional Filter Banks [J]. Applied Mathematics and Computation, 2008, 205(2): 517-524.

[12] Liu W. Y. Image Coding and Specific VLSI Design Based on Wavelet Transform [M]. Beijing: Publishing house of electronics industry, 2006.

[13] Donoho D. L., Johnstone I. M. Ideal Spatial Adaptation via Wavelet Shrinkage [J]. Biometrika, 1994, 81: 425-455. 